\title{
TOA Estimation Based on Forward and Backward Smoothing MUSIC in Frequency Domain
}

\author{
Bin $\mathrm{Ba}$, Yun Long Wang, Na E Zheng \& Han Ying Hu \\ Zhengzhou Institute of Information Science and Technology, Zhengzhou, 450002, China
}

Keywords: smoothing, MUSIC, TOA.

\begin{abstract}
Under the condition of single snapshot, multiple signal classification (MUSIC) time of arrival (TOA) estimation algorithm improves its performance by forward and backward smoothing in frequency domain. Its ability to distinguish multipath is strong. However, this method is introduced from smoothing in spatial smoothing. And the derivation and the performance analysis are not given. For this problem, the derivation and performance analysis of MUSIC TOA estimation based on forward and backward smoothing in frequency domain is introduced in this paper. The full-rank conditions of autocorrelation matrix of multipath component fading coefficient are deduced. At the same time, estimation precision of noise subspace is higher after data is preprocessed by forward and backward smoothing. Simulation results show that the algorithm has better ability to distinguish multipath under situations of single snapshot. The algorithm has strong robustness and its performance is better than MUSIC TOA estimation algorithm of forward smoothing in frequency domain.
\end{abstract}

\section{Introduction}

With the rapid development of location services and the next generation of location-aware wireless network, location technology becomes more and more important. The demand of military, commercial and emergency service has improved the positioning accuracy of wireless positioning system greatly. On the other hand, the location information is helpful for the performance of wireless communication system. Mobile communications operators can provide different services for the users according to the position of users. The technology of orthogonal frequency division multiplexing (OFDM) with its advantages of high band efficiency, has been widely used in wireless communication system. Therefore, the location technology of OFDM wireless communication has been widely concerned.

Time of arrival (TOA) estimation in OFDM wireless communication system is one of the important contents of the high precision positioning. In the indoor and city environment, the condition of multipath and the complexity of wireless communication affect the precision of TOA estimation. Super resolution technology with high precision is applied to the TOA estimation, such as multiple signal classification (MUSIC) algorithm [1-3], the estimation of signal parameters via rotational invariance technique (ESPRIT) [4], Root-MUSIC [5], propagator method (PM) [6] and so on, which has high precision of TOA estimation in a large number of snapshots.

In this paper, autocorrelation matrix of multipath component fading coefficient is fully ranked by forward and backward smoothing in frequency domain. Limiting conditions are given. At the same time, forward and backward smoothing is equivalent to increasing the samples of channel frequency response and improves the estimation accuracy of autocorrelation matrix of channel frequency response. Then, it improves the estimation accuracy and the robustness of the MUSIC algorithm.

\section{Signal model and MUSIC algorithm for TOA}

\section{Signal model}

In the OFDM wireless communication system, the impulse response of wireless multipath channel is

$$
h(t)=\sum_{i=0}^{L_{p}-1} \alpha_{i} \delta\left(t-\tau_{i}\right), \quad 0 \leq i \leq L_{P}-1,
$$


where $L_{P}$ is the number of multipath components. Let $\alpha_{i}=\left|\alpha_{i}\right| e^{j \theta_{i}}$ represent the fading coefficients of $i$ th multipath component and $\tau_{i}$ represent Propagation delay of $i$ th multipath component. Propagation delay is ordered according to the ascending order. $\tau_{0}$ is the propagation delay of sight pathway, namely parameters of TOA estimation. $\delta$ represents impulse function.

The channel frequency domain response estimation can be written as matrix form

$$
\hat{\boldsymbol{H}}=\boldsymbol{H}+\boldsymbol{n}=\boldsymbol{D} \boldsymbol{\alpha}+\boldsymbol{n},
$$

where $\boldsymbol{D}$ is the matrix composed by the steering vector.

\section{MUSIC algorithm for TOA}

Make eigenvalue decomposition of the autocorrelation matrix in (2)

$$
\boldsymbol{R}=E\left[\hat{\boldsymbol{H}} \hat{\boldsymbol{H}}^{\mathrm{H}}\right]=\boldsymbol{D} \boldsymbol{R}_{\alpha} \boldsymbol{D}^{\mathrm{H}}+\sigma^{2} \boldsymbol{I} .
$$

where $\boldsymbol{R}_{\alpha}=E\left[\alpha \alpha^{\mathrm{H}}\right]$ is the autocorrelation matrix of multipath component fading coefficient. Because $\alpha$ is a constant and its phase satisfies uniform distribution $(0,2 \pi) \cdot L_{P} \times L_{P}$ dimensional matrix $\operatorname{rank}\left(\boldsymbol{R}_{\alpha}\right)=L_{P}$ is a non-singular matrix. The rank of Vandermonde matrix $\boldsymbol{D}$ is $\operatorname{rank}(\boldsymbol{D})=L_{P}$. So $\operatorname{rank}\left(\boldsymbol{D} \boldsymbol{R}_{\alpha} \boldsymbol{D}^{\mathrm{H}}\right)=L_{P}$. I is a identity matrix.

Making spectral decomposition of $\boldsymbol{R}$, we can obtain

$$
\boldsymbol{R}=\sum_{i=0}^{L_{p}-1} \lambda_{i} \boldsymbol{u}_{i} \boldsymbol{u}_{i}^{\mathrm{H}}+\sum_{i=L_{p}}^{K-1} \lambda_{i} \boldsymbol{u}_{i} \boldsymbol{u}_{i}^{\mathrm{H}}=\boldsymbol{U}_{S} \boldsymbol{\Lambda}_{S} \boldsymbol{U}_{S}^{\mathrm{H}}+\boldsymbol{U}_{N} \boldsymbol{\Lambda}_{N} \boldsymbol{U}_{N}^{\mathrm{H}} \cdot
$$

It is easy to find that eigenvalues of $\boldsymbol{R}$ satisfies the following distribution,

$$
\lambda_{0} \geq \lambda_{1} \geq \cdots \geq \lambda_{L_{p}-1} \geq \lambda_{L_{p}}=\lambda_{L_{p}+1}=\cdots=\lambda_{K-1}=\sigma^{2} .
$$

Diagonal matrix $\Lambda_{S}=\operatorname{diag}\left[\lambda_{0}, \lambda_{1}, \cdots, \lambda_{L_{p}-1}\right]$, diagonal matrix $\Lambda_{N}=\operatorname{diag}\left[\lambda_{L_{p}}, \lambda_{L_{p}+1}, \cdots, \lambda_{K-1}\right]$. The liner subspace $\operatorname{span}\left(\boldsymbol{U}_{S}\right)$ formed by $\boldsymbol{U}_{S}=\left[\boldsymbol{u}_{0}, \boldsymbol{u}_{1}, \cdots, \boldsymbol{u}_{L_{p}-1}\right]$ which is called signal subspace and the subspace $\operatorname{span}\left(\boldsymbol{U}_{N}\right)$ formed by $\boldsymbol{U}_{N}=\left[\boldsymbol{u}_{L_{p}}, \boldsymbol{u}_{L_{p}+1}, \cdots, \boldsymbol{u}_{K-1}\right]$ is called noise subspace.

According to the literature

$$
\boldsymbol{d}^{\mathrm{H}}\left(\tau_{i}\right) \boldsymbol{U}_{N}=\mathbf{0}, \quad 0 \leq i \leq L_{P}-1,
$$

Pseudo-spectrum of MUSIC algorithm can be expressed as

$$
P_{\text {MUSIC }}(\tau)=\frac{1}{\left\|\boldsymbol{d}^{\mathrm{H}}(\tau) \boldsymbol{U}_{N}\right\|^{2}} \text {. }
$$

Because $\boldsymbol{d}^{\mathrm{H}}\left(\tau_{i}\right) \boldsymbol{U}_{N}=\mathbf{0}, P_{\text {MUSIC }}\left(\tau_{i}\right)$ is the pseudo-spectral of MUSIC algorithm.

\section{TOA estimation}

TOA estimation based on MUSIC algorithm has good estimation accuracy and robustness under condition of multi-snapshots. In a single snapshot, the accuracy of TOA estimation can be increased by pre-processing the sampling points in frequency domain.

In a single snapshot, autocorrelation matrix of the fading coefficients of multipath component $\boldsymbol{R}_{\alpha}=\boldsymbol{\alpha} \boldsymbol{\alpha}^{\mathrm{H}}$ and $\operatorname{rank}\left(\boldsymbol{R}_{\alpha}\right)=1$, then

$$
\boldsymbol{R}=\boldsymbol{D} \boldsymbol{\alpha} \boldsymbol{\alpha}^{\mathrm{H}} \boldsymbol{D}^{\mathrm{H}}+\sigma^{2} \boldsymbol{I} \square \boldsymbol{b} \boldsymbol{b}^{\mathrm{H}}+\sigma^{2} \boldsymbol{I},
$$

where $\boldsymbol{b}=\boldsymbol{D} \boldsymbol{\alpha}, \lambda_{1}=\lambda_{2}=\cdots=\lambda_{K-1}=\sigma^{2}$, therefore

$$
\boldsymbol{b}^{\mathrm{H}}\left[\boldsymbol{u}_{1}, \boldsymbol{u}_{2}, \cdots, \boldsymbol{u}_{K-1}\right]=\mathbf{0} \text {. }
$$

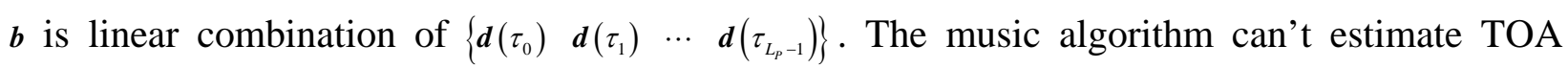
correctly through (9). In order to estimate TOA correctly, $\boldsymbol{R}_{\alpha}$ must be a non-singular matrix. So make forward and backward smoothing in frequency domain, as is shown in Fig1. 


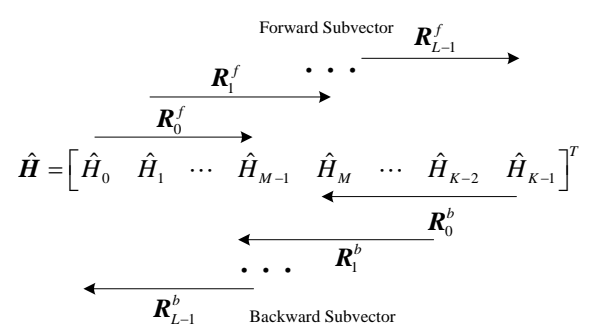

Fig.1: Forward and backward smoothing in frequency domain.

\section{Forward frequency smoothing}

Divide $\hat{\boldsymbol{H}}$ with the length of $K$ into $L$ forward subvector with the length of $M$

$$
\hat{\boldsymbol{H}}_{l}^{f} \square\left[\begin{array}{llll}
\hat{H}_{l} & \hat{H}_{l+1} & \cdots & \hat{H}_{l+M-1}
\end{array}\right]^{T}=\boldsymbol{D} \boldsymbol{B}^{l} \boldsymbol{\alpha}+\boldsymbol{n}_{l}, \quad 0 \leq l \leq L-1,
$$

where sliding cycle number $L=K-M+1$. Autocorrelation matrix of the forward subvector can be divided into signal subspace and noise subspace, and the dimension of noise subspace is at least 1 (that is $\left.M \geq L_{P}+1\right) . B^{l}$ represents the $l$ th $L_{P} \times L_{P}$ diagonal matrix and

$$
\boldsymbol{B}=\operatorname{diag}\left\{v_{0}, v_{1}, \cdots, v_{L_{P}-1}\right\}, \quad v_{i}=\exp \left(-j \frac{2 \pi}{T} \tau_{i}\right), \quad 0 \leq i \leq L_{P}-1 .
$$

The autocorrelation matrix of $\hat{\boldsymbol{H}}_{l}^{f}$ is

$$
\boldsymbol{R}_{l}^{f}=E\left[\hat{\boldsymbol{H}}_{l}^{f}\left(\hat{\boldsymbol{H}}_{l}^{f}\right)^{\mathrm{H}}\right]=\boldsymbol{D} \boldsymbol{B}^{l} \boldsymbol{R}_{\alpha}\left(\boldsymbol{B}^{l}\right)^{\mathrm{H}} \boldsymbol{D}^{\mathrm{H}}+\sigma^{2} \boldsymbol{I} .
$$

The autocorrelation matrix of forward frequency smoothing is

$$
\boldsymbol{R}^{f}=\frac{1}{L} \sum_{l=0}^{L-1} \boldsymbol{R}_{l}^{f}=\boldsymbol{D} \boldsymbol{R}_{\alpha}^{f} \boldsymbol{D}^{\mathrm{H}}+\sigma^{2} \boldsymbol{I} .
$$

The autocorrelation matrix $\boldsymbol{R}_{\alpha}^{f}$ of the fading coefficients of forward frequency smoothing multipath component is

$$
\boldsymbol{R}_{\alpha}^{f} \square \frac{1}{L} \sum_{l=0}^{L-1} \boldsymbol{B}^{l} \boldsymbol{R}_{\alpha}\left(\boldsymbol{B}^{l}\right)^{\mathrm{H}}=\frac{1}{L} \boldsymbol{C} \boldsymbol{C}^{\mathrm{H}},
$$

where

$$
\begin{aligned}
& \boldsymbol{C}=\left[\boldsymbol{\alpha}, \boldsymbol{B} \boldsymbol{\alpha}, \boldsymbol{B}^{2} \boldsymbol{\alpha}, \cdots, \boldsymbol{B}^{L-1} \boldsymbol{\alpha}\right] \\
& =\left[\begin{array}{cccc}
\alpha_{0} & 0 & \cdots & 0 \\
0 & \alpha_{1} & \cdots & 0 \\
0 & 0 & \ddots & 0 \\
0 & 0 & \cdots & \alpha_{L_{p}-1}
\end{array}\right]\left[\begin{array}{ccccc}
1 & v_{0} & v_{0}^{2} & \cdots & v_{0}^{L-1} \\
1 & v_{1} & v_{1}^{2} & \cdots & v_{1}^{L-1} \\
\vdots & \vdots & \vdots & & \vdots \\
1 & v_{L_{p}-1} & v_{L_{p}-1}^{2} & \cdots & v_{L_{p}-1}^{L-1}
\end{array}\right] \square \boldsymbol{G V}
\end{aligned}
$$

When $L \geq L_{P}$, from formula (14) and (15), the rank of Vandermonde matrix is $\operatorname{rank}(\boldsymbol{V})=L_{P}$. Because $\operatorname{rank}(\boldsymbol{G})=L_{P}$, then $\operatorname{rank}(\boldsymbol{C})=L_{P}$. Therefore, $\operatorname{rank}\left(\boldsymbol{R}_{\alpha}^{f}\right)=\operatorname{rank}\left(\frac{1}{2 L} \boldsymbol{C} \boldsymbol{C}^{\mathrm{H}}\right)=L_{P}\left(\boldsymbol{R}_{\alpha}^{f}\right.$ is fully ranked $)$.

$L=K-M+1$ can be concluded by smoothing in frequency domain. $M \geq L_{P}+1$ can be get by (10). From the three formulas, we deduce

$$
\left\{\begin{array}{c}
L \geq L_{P} \\
L=K-M+1 \Rightarrow K \geq 2 L_{P} . \\
M \geq L_{P}+1
\end{array}\right.
$$

Therefore, $\boldsymbol{R}_{\alpha}^{f}$ is fully ranked when the minimum of $K$ is $2 L_{P}$.

\section{Backward frequency smoothing}

As Fig 1 shows, make $L$ backward subvectors with the length of $M$,

$$
\hat{\boldsymbol{H}}_{l}^{b} \square\left[\hat{H}_{K-l-1}^{*}, \hat{H}_{K-l-2}^{*}, \cdots, \hat{H}_{K-l-M}^{*}\right]^{T}=\boldsymbol{D} \boldsymbol{B}^{l}\left(\boldsymbol{B}^{K-1} \boldsymbol{\alpha}\right)^{*}+\boldsymbol{n}_{l}^{*}, \quad 0 \leq l \leq L-1 .
$$

The autocorrelation matrix of $l$ th $L$ backward sub vector is

$$
\boldsymbol{R}_{l}^{b}=E\left[\hat{\boldsymbol{H}}_{l}^{b}\left(\hat{\boldsymbol{H}}_{l}^{b}\right)^{\mathrm{H}}\right]=\boldsymbol{D} \boldsymbol{B}^{l} \boldsymbol{R}_{\tilde{\alpha}}\left(\boldsymbol{B}^{l}\right)^{\mathrm{H}} \boldsymbol{D}^{\mathrm{H}}+\sigma^{2} \boldsymbol{I},
$$

where

$$
\boldsymbol{R}_{\tilde{\alpha}} \square \boldsymbol{B}^{-(K-1)} E\left[\boldsymbol{\alpha}^{*} \boldsymbol{\alpha}^{T}\right]\left(\boldsymbol{B}^{-(K-1)}\right)^{\mathrm{H}}=\boldsymbol{B}^{-(K-1)} \boldsymbol{R}_{\alpha}^{*}\left(\boldsymbol{B}^{-(K-1)}\right)^{\mathrm{H}} .
$$

The autocorrelation matrix of backward frequency smoothing is 


$$
\boldsymbol{R}^{b}=\frac{1}{L} \sum_{l=0}^{L-1} \boldsymbol{R}_{l}^{b}=\boldsymbol{D} \boldsymbol{R}_{\alpha}^{b} \boldsymbol{D}^{\mathrm{H}}+\sigma^{2} \boldsymbol{I}
$$

$\boldsymbol{R}_{\tilde{\alpha}}$ is

where

$$
\boldsymbol{R}_{\tilde{\alpha}}=\boldsymbol{q} \boldsymbol{q}^{\mathrm{H}}
$$

$$
\boldsymbol{\beta}=\left[\beta_{0}, \beta_{1}, \cdots, \beta_{L_{p}-1}\right]^{\mathrm{T}}, \quad \beta_{i}=\alpha_{i}^{*} v_{i}^{-(K-1)}, \quad 0 \leq i \leq L_{P}-1 .
$$

The autocorrelation matrix $\boldsymbol{R}_{\alpha}^{b}$ of the fading coefficients of backward frequency smoothing multipath component is

$$
\boldsymbol{R}_{\alpha}^{b} \square \frac{1}{L} \sum_{l=0}^{L-1} \boldsymbol{B}^{l} \boldsymbol{R}_{\tilde{\alpha}}\left(\boldsymbol{B}^{l}\right)^{\mathrm{H}}=\frac{1}{L} \boldsymbol{E} \boldsymbol{E}^{\mathrm{H}},
$$

where

$$
\begin{gathered}
\boldsymbol{E}=\left[\boldsymbol{\beta}, \boldsymbol{B} \boldsymbol{\beta}, \boldsymbol{B}^{2} \boldsymbol{\beta}, \cdots, \boldsymbol{B}^{L-1} \boldsymbol{\beta}\right] \square \boldsymbol{F} \boldsymbol{V}, \\
\boldsymbol{F}=\operatorname{diag}\left\{\beta_{0}, \beta_{1}, \cdots, \beta_{L_{p}-1}\right\} .
\end{gathered}
$$

\section{Forward and backward smoothing}

The autocorrelation matrix $\tilde{\boldsymbol{R}}$ of forward and backward frequency smoothing is

$$
\tilde{\boldsymbol{R}}=\frac{\boldsymbol{R}^{f}+\boldsymbol{R}^{b}}{2}=\boldsymbol{D} \tilde{\boldsymbol{R}}_{\alpha} \boldsymbol{D}^{\mathrm{H}}=\boldsymbol{D}\left[\frac{1}{2 L}\left(\boldsymbol{C} \boldsymbol{C}^{\mathrm{H}}+\boldsymbol{E} \boldsymbol{E}^{\mathrm{H}}\right)\right] \boldsymbol{D}^{\mathrm{H}}+\sigma^{2} I,
$$

where

$$
\begin{gathered}
\tilde{\boldsymbol{R}}_{\boldsymbol{\alpha}}=\frac{1}{2 L}\left(\boldsymbol{C C}^{\mathrm{H}}+\boldsymbol{E} \boldsymbol{E}^{\mathrm{H}}\right)=\frac{1}{2 L} \boldsymbol{Q} \boldsymbol{Q}^{\mathrm{H}}, \\
\boldsymbol{Q}=\left[\boldsymbol{\alpha}, \boldsymbol{B} \boldsymbol{\alpha}, \boldsymbol{B}^{2} \boldsymbol{\alpha}, \cdots, \boldsymbol{B}^{L-1} \boldsymbol{\alpha}, \boldsymbol{\beta}, \boldsymbol{B} \boldsymbol{\beta}, \boldsymbol{B}^{2} \boldsymbol{\beta}, \cdots, \boldsymbol{B}^{L-1} \boldsymbol{\beta}\right], \\
=[\boldsymbol{G} \boldsymbol{V} \mid \boldsymbol{F} \boldsymbol{V}]=\boldsymbol{G}[\boldsymbol{V} \mid \boldsymbol{H} \boldsymbol{V}] \square \boldsymbol{G G}_{0} \\
\boldsymbol{H}=\operatorname{diag}\left\{\gamma_{0}, \gamma_{1}, \cdots, \gamma_{L_{P}-1}\right\}, \quad \gamma_{i}=\beta_{i} / \alpha_{i}, \quad i=0,1, \cdots, L_{P}-1 .
\end{gathered}
$$

Assume that $2 L \geq L_{P} \geq L, a_{i}=\left|a_{i}\right| e^{j \theta_{i}}, v_{i}=\exp \left(-j \frac{2 \pi}{T} \tau_{i}\right)$ and $\delta_{i}=a_{i}^{*} v_{i}^{-(N-1)}$ are fed into $\varepsilon_{i}=\delta_{i} / \alpha_{i}$, then

$$
\gamma_{i}=\beta_{i} / \alpha_{i}=\alpha_{i}^{*} v_{i}^{(K-1)} / \alpha_{i}=\exp \left[j \frac{2 \pi}{T}(K-1) \tau_{i}-2 \theta_{i}\right], \quad 0 \leq i \leq L_{P}-1 .
$$

Because $\tau_{i}$ is related with signal propagation environment, $\theta_{i}$ satisfies uniform distribution $\operatorname{in}(0,2 \pi)$. Then

$$
\gamma_{i} \neq \gamma_{j} ; \quad i=0,1, \cdots, L-1 ; \quad j=L, L+1, \cdots, L_{P}-1 .
$$

$\boldsymbol{G}_{0}$ is a Vandermonde matrix whose columns are not equal. Because $2 L \geq L_{P}$, then $\operatorname{rank}\left(\boldsymbol{G}_{0}\right)=L_{P}$. Due to $\operatorname{rank}(\boldsymbol{G})=L_{P}$, then $\operatorname{rank}(\boldsymbol{Q})=L_{P}$. So $\operatorname{rank}\left(\tilde{\boldsymbol{R}}_{\alpha}\right)=\operatorname{rank}\left(\frac{1}{2 L} \mathbf{Q} \mathbf{Q}^{\mathrm{H}}\right)=L_{P}\left(\tilde{\boldsymbol{R}}_{\alpha}\right.$ is fully ranked $)$.

$M \geq L_{P}+1$ can be concluded from (14). From the three formulas, we deduce

$$
\left\{\begin{array}{c}
2 L \geq L_{P} \\
L=K-M+1 \Rightarrow K \geq 3 L_{P} / 2 . \\
M \geq L_{P}+1
\end{array}\right.
$$

\section{Performance analysis}

Through the above derivation, The full-rank conditions of autocorrelation matrix of modified multipath component fading coefficient were deduced. When $\tilde{\boldsymbol{R}}_{\alpha}$ is fully ranked, the dimension of $\hat{\boldsymbol{H}}$ is at least ${ }^{3 L_{P} / 2}$. When $\boldsymbol{R}_{\alpha}^{f}$ is fully ranked, the dimension of $\hat{\boldsymbol{H}}$ is at least ${ }^{2 L_{P}}{ }^{3 L_{P} / 2}$. When the dimension of $\hat{\boldsymbol{H}}$ is certain, the statistical sample of $\boldsymbol{R}^{f}$ is $L$, the statistical sample of $\tilde{\boldsymbol{R}}$ is $2 L$. Because the precision of autocorrelation matrix of frequency smoothing is limited to the statistical sample, the statistical performance of $\tilde{\boldsymbol{R}}$ is better than $\boldsymbol{R}^{f}$, and the corresponding MUSIC algorithm is more stable.

Besides, the resolution of MUSIC algorithm is affected by bandwidth. Thus, $M$ decides the 
bandwidth. Therefore, the value of $M$ is a compromise of resolution and stability. Under the same conditions, because the statistical performance of $\tilde{\boldsymbol{R}}$ is better, the performance of the MUSIC algorithm of forward and backward frequency smoothing is better.

\section{Simulation results}

The good resolution of TOA is embodied on the pseudo spectrum where there is sharp peak on the arrival time of multipath whose two multipath components is close, but the amplitude of curve should be as low as possible on the arrival time of non-multipath, especially the middle of two multipath components' arrival time. So define two multipath components whose arrival time is $\tau_{0}$, $\tau_{1}$. In an experiment, if there are two "effective peak" whose estimation time is $\hat{\tau}_{0}, \hat{\tau}_{1}$ in normalized pseudo spectrum and the estimate time meets $\left|\hat{\tau}_{0}-\tau_{0}\right|+\left|\hat{\tau}_{1}-\tau_{1}\right|<\left|\hat{\tau}_{0}-\hat{\tau}_{1}\right|$, the multipath is distinguished successfully. In this paper, the "effective peak" is the peak whose top is taller than the bottom beside the peak at least $3 \mathrm{~dB}$ in the normalized pseudo spectrum.

Assume that the snapshot is 1 . The arrival time of two multipath is $50 \mathrm{~ns}$ and $60 \mathrm{~ns}$. SNR=10dB, $M=40$.

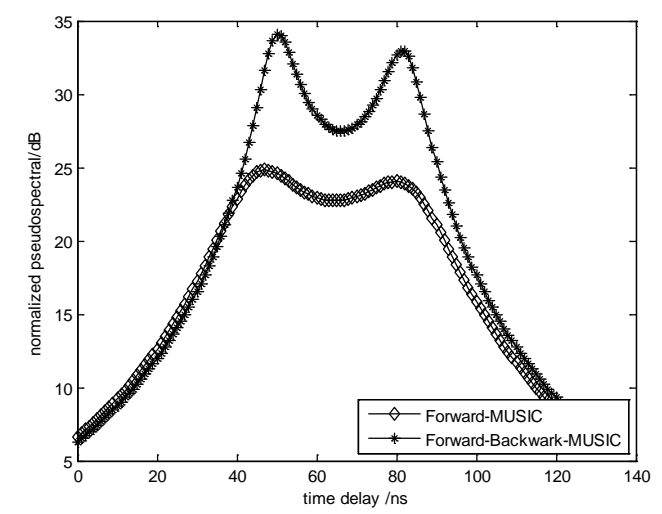

Fig.2: Resolution capability comparison of the Forward MUSIC and Forward Backward MUSIC.

From Fig 2, forward and backward frequency smoothing MUSIC algorithm has better ability to distinguish multipath. Compared with forward and backward smoothing pre-processing, forward and backward smoothing pre-processing has twice of the samplings. Therefore, autocorrelation matrix of forward and backward frequency smoothing MUSIC algorithm has better ability to distinguish multipath.

\section{Conclusions}

Single-snapshot MUSIC algorithm could effectively improve the estimation accuracy of MUSIC time delay estimation algorithm by forward frequency smoothing. But there are no full-rank conditions of autocorrelation matrix of multipath component fading coefficient in the existing literature. For the situation, the full-rank conditions $\left(K \geq 2 L_{P}\right)$ of data pre-processing by forward frequency smoothing and the full-rank conditions $\left(K \geq 3 L_{P} / 2\right)$ of data pre-processing by forward and backward frequency smoothing in this paper. Data pre-processing by forward and backward frequency smoothing has more samples and improves the estimation accuracy of the noise subspace, so it improves the estimation accuracy of TOA.

\section{References}

[1] Li X., \& Pahlavan K., Super-resolution TOA estimation with diversity for indoor geolocation. IEEE Trans. Wireless Commun., 3(1), pp.224-234, 2004. 
[2] Li X., Ma X., Yan S., \& Hou C., Super-resolution time delay estimation for narrowband signal. IET Radar Sonar \& Navigation, 6(8), pp.781-787, 2012.

[3] Zhang X., Feng G., \& Xu D., Blind direction of angle and time delay estimation algorithm for uniform linear array employing multi-invariance music. Progress In Electromagnetics Research Letters, 13, pp.11-20, 2010.

[4] Oh D., Kim S., Yoon S. H., \& Chong J. W., Two-dimensional ESPRIT-like shift-invariant TOA estimation algorithm using multi-band chirp signals robust to carrier frequency offset. IEEE Trans. Wireless Commun., 12(7), pp.3130-3139, 2013.

[5] Wang F. Q., Zhang X. F., \& Wang F., Root-MUSIC-based joint TOA and DOA estimation in IR-UWB. Journal on Communications, 35(2), pp.137-145, 2014.

[6] Wang F. Q., \& Zhang X. F., Improved propagator method-based joint TOA and DOA estimation in impulse radio ultra wideband. Journal of Electronics \& Information Technology, 35(12), pp.2954-2959, 2013. 